\title{
Effect of Repetitive Lysine-Tryptophan Motifs on the Eukaryotic Membrane
}

\author{
Ramamourthy Gopal ${ }^{1}$, Jong Kook Lee ${ }^{1}$, Jun Ho Lee ${ }^{2}$, Young Gwon Kim ${ }^{2}$, Gwang Chae Oh ${ }^{3}$, \\ Chang $\mathrm{Ho} \mathrm{Seo}^{3}$ and Yoonkyung Park ${ }^{1,2, *}$
}

1 Research Center for Proteineous Materials, Chosun University, Gwangju 501-759, Korea;

E-Mails: ramagopa@gmail.com (R.G.); sea19669@hanmail.net (J.K.L.)

2 Department of Biotechnology, Chosun University, Gwangju 501-759, Korea;

E-Mails: juno6267@hanmail.net (J.H.L.); kyg1022@hanmail.net (Y.G.K.)

3 Department of Bioinformatics, Kongju National University, Kongju 314-701, Korea;

E-Mails: gcoh@gjtp.or.kr (G.C.O.); chseo@kongju.ac.kr (C.H.S.)

* Author to whom correspondence should be addressed; E-Mail: y_k_park@chosun.ac.kr;

Tel.: +82-62-230-6854; Fax: +82-62-225-6758.

Received: 27 November 2012; in revised form: 14 January 2013 / Accepted: 15 January 2013 /

Published: 22 January 2013

\begin{abstract}
In a previous study, we synthesized a series of peptides containing simple sequence repeats, $(\mathrm{KW})_{n}-\mathrm{NH}_{2}(n=2,3,4$ and 5) and determined their antimicrobial and hemolytic activities, as well as their mechanism of antimicrobial action. However, $(\mathrm{KW})_{5}$ showed undesirable cytotoxicity against RBC cells. In order to identify the mechanisms behind the hemolytic and cytotoxic activities of $(\mathrm{KW})_{5}$, we measured the ability of these peptides to induce aggregation of liposomes. In addition, their binding and permeation activities were assessed by Trp fluorescence, calcein leakage and circular dichrorism using artificial phospholipids that mimic eukaryotic liposomes, including phosphatidylcholine (PC), PC/sphingomyelin (SM) $(2: 1, w / w)$ and PC/cholesterol $(\mathrm{CH})(2: 1, w / w)$. Experiments confirmed that only $(\mathrm{KW})_{5}$ induced aggregation of all liposomes; it formed much larger aggregates with PC:CH $(2: 1, w / w)$ than with PC or PC:SM $(2: 1, w / w)$. Longer peptide $(\mathrm{KW})_{5}$, but not $(\mathrm{KW})_{3}$ or $(\mathrm{KW})_{4}$, strongly bound and partially inserted into $\mathrm{PC}$ : $\mathrm{CH}$ compared to $\mathrm{PC}$ or $\mathrm{PC}: \mathrm{SM}(2: 1, w / w)$. Calcein release experiments showed that $(\mathrm{KW})_{5}$ induced calcein leakage from the eukaryotic membrane. Greater calcein leakage was induced by $(\mathrm{KW})_{5}$ from PC:CH than from PC:SM $(2: 1, w / w)$ or PC, whereas $(\mathrm{KW})_{4}$ did not induce calcein leakage from any of the liposomes. Circular dichroism measurements indicated that $(\mathrm{KW})_{5}$ showed higher conformational transition compared to $(\mathrm{KW})_{4}$ due to peptide-liposome interactions.
\end{abstract}


Taken together, our results suggest that $(\mathrm{KW})_{5}$ reasonably mediates the aggregation and permeabilization of eukaryotic membranes, which could in turn explain why $(\mathrm{KW})_{5}$ displays efficient hemolytic activity.

Keywords: $(\mathrm{KW})_{5}$; hemolytic peptide; eukaryotic membrane; phosphatidylcholine; cholesterol; sphingomyelin; aggregation

\section{Introduction}

Native and synthetic antimicrobial peptides (AMPs) exhibited a broad spectrum of activities against antibiotic resistant bacterial strains, including cancer cells [1]. Moreover, AMPs constitute key parts of the human and mammalian innate immune systems. In addition, AMPs are also involved in multiple defensive roles, such as regulating the inflammatory response and chemo-attracting cells of the adaptive immune system to wound/infection sites, binding and neutralizing lipopolysaccharides and promote re-epithelialization and wound closure in human skin against bacterial infections [2,3]. However, synthetic peptides utility is currently limited to topical application, because of their cytotoxicity against the eukaryotic cell membranes.

Prokaryotic and eukaryotic cell membranes are mainly composed of phospholipids that differentiate their compositions [4]. It is well known that eukaryotic cell membranes are mainly composed of electrically neutral zwitterionic phospholipids, including phosphatidylcholine (PC), cholesterol $(\mathrm{CH})$ and sphingomyelin (SM) [5]. Among these three phospholipids, the percentage of PC in the outer leaflets of eukaryotic membranes is higher than that of $\mathrm{CH}$ or SM [6]. Indeed, the outer leaflets of eukaryotic membranes are composed of lipids with no net charge. Accordingly, the small amount of negatively charged lipids is essentially located in the inner leaflet [7]. In contrast, bacterial membranes (e.g., in E. coli) lack $\mathrm{CH}$, contain phosphatidylethanolamine as their most common zwitterionic lipid and are rich in anionic lipids, such as phosphatidylglycerol, and cardiolipin in their outer membranes [8]. This differentiation in phospholipid content between prokaryotic and eukaryotic membranes promotes selectivity by AMPs $[9,10]$. This selectivity of AMPs towards bacterial plasma membranes is very important for the design of novel antibiotic peptides with low cytotoxicity. However, many AMPs are non-selective between mammalian and microbial cells (e.g., pardaxin [11], melittin [12] and cathelicidins [13]). Therefore, various studies have attempted to determine the parameters that are responsible for AMP toxicity. It has been determined that high helicity, hydrophobicity and amphipathicity are correlated with an increase in eukaryotic cell toxicity [14]. Recent studies have also shown that aggregation/oligomeration of cationic antimicrobial peptides (cAMPs) is another parameter that is important for pore formation, regardless of the mechanism of cytotoxicity $[15,16]$. Accordingly, efforts have been undertaken to improve the selectivity of cAMPs, including sequence modification, to optimize their physiochemical parameters [17-20]. At present, short AMPs that minimize damage to host cells or tissue appear to be the most promising candidates for large-scale production [21,22]. In addition, the large size of AMPs is a hindrance due to high manufacturing costs. In response, selective short AMPs are currently being developed based on their amino acid composition, charge, hydrophobicity and length [21-25]. Specifically, lysine (K) or arginine (R)-tryptophan (W or Trp) or 
isoleucine (I)-phenylalanine (F) motifs have been designed for bacterial membrane selectivity $[22,26]$. However, KW, RIF or RW series peptides show increased hemolytic activity and hydrophobicity with higher chain length [22,26]. In a separate study using a de novo designed alpha helical HDP, high hydrophobicity was found to correlate with strong hemolytic activity [27]. However, there is insufficient data to support this claim. While hemolytic activity correlates with hydrophobicity values, there could be other factors affecting hemolytic activity. Therefore, we investigated the effects of peptide on model vesicles composed of a mixture of $\mathrm{PC}, \mathrm{CH}$ and $\mathrm{SM}$ in order to determine their molecular mechanisms, since these three lipids are major constituents of most mammalian plasma membranes. It is also important to note that $\mathrm{CH}$ effectively suppressed the toxicity of AMPs against the eukaryotic membrane by preventing peptide induced eukaryotic membrane disruption via a barrel stave mechanism or other mechanism [28,29]. Also, a variety of biophysical approaches have reported mechanisms of membrane permeation/disruption activities of AMPs, including AMPs-induced liposome aggregation [30-33]. However, we believe this study will be useful in understanding the role of PC, SM and CH on hemolytic activity of AMPs. We found that only $(\mathrm{KW})_{5}$ showed a strong interaction towards PC, PC:SM $(2: 1, w / w)$ and PC:CH $(2: 1, w / w)$ of the erythrocyte membrane. Moreover, $(\mathrm{KW})_{5}$ induced aggregation/permeabilization of eukaryotic membranes.

\section{Results and Discussion}

Several studies have clearly shown that AMPs demonstrate activity against negatively charged bacterial membranes, whereas AMPs do not show strong activity towards zwitterionic eukaryotic membranes, suggesting non-activity against human cells [34-40]. Similarly, $(\mathrm{KW})_{4}$ has shown strong selectivity against bacterial over eukaryotic membranes based on membrane interactions. However, when chain length exceeds the optimum number of 10 residues, $(\mathrm{KW})_{5}$ shows interactions with both bacterial and eukaryotic membranes, resulting in antibacterial activity with cytoxicity towards eukaryotic cells [26]. In the present study, we investigated the interactions of $(\mathrm{KW})_{n}$ peptides with eukaryotic membranes to determine the increased hemolytic mechanism of $(\mathrm{KW})_{5}$ compared to $(\mathrm{KW})_{4}$.

\subsection{Liposomes Aggregation}

Liposome aggregation showed peptide-lipid interactions as previously reported [26,41]. We measured changes in liposome turbidity in order to determine the effects of $(\mathrm{KW})_{n}$ peptides on the sizes of PC, PC:SM $(2: 1, w / w)$ and PC:CH $(2: 1, w / w)$ vesicles (Figure 1). We found that $(\mathrm{KW})_{2},(\mathrm{KW})_{3}$ and $(\mathrm{KW})_{4}$ did not induce liposome aggregation towards PC, PC:SM $(2: 1, w / w)$ or PC:CH $(2: 1, w / w)$ vesicles, even at larger peptide concentrations. However, our results showed that $(\mathrm{KW})_{5}$ was able to induce aggregation of liposomes. In addition, $(\mathrm{KW})_{5}$ induced larger aggregation of $\mathrm{PC}: \mathrm{CH}$ than either $100 \%$ PC or PC:SM $(2: 1, w / w)$. This could be attributed to the membrane behavior of $\mathrm{CH}$ leading to enhanced peptide accumulation in the hydrophobic core of its bilayers, resulting in increased liposome aggregation. It was reported that $(\mathrm{KW})_{5}$ is soluble and weakly self-aggregates in PBS, as determined from Trp fluorescence spectra, showing W-residues to be in a less polar environment $\left(\lambda_{\max }=342\right)$ [26]. Other studies have proposed that a peptide's aggregation state in solution might affect eukaryotic membranes, resulting in cytotoxicity against RBCs $[42,43]$. This is in accordance with previous data on the hemolytic activity of $(\mathrm{KW})_{5}$ peptide [26]. 
Figure 1. Large unilamellar vesicles (LUV) aggregation. Solutions containing various concentrations of peptide were added to a suspension of $400 \mu \mathrm{M}$ PC (diamonds), PC:SM $(2: 1, w / w)$ (squares) and PC:CH $(2: 1, w / w)$ (triangles), after which aggregation was monitored based on changes in the absorbance of LUVs at $405 \mathrm{~nm}$. (A) $(\mathrm{KW})_{2}$; (B) $(\mathrm{KW})_{3}$; (C) $(\mathrm{KW})_{4} ;(\mathbf{D})(\mathrm{KW})_{5}$.

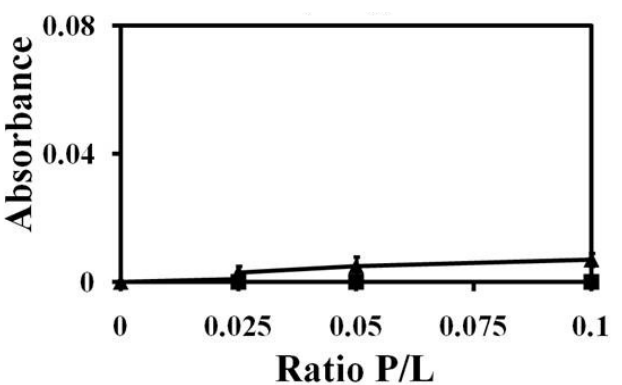

(A)

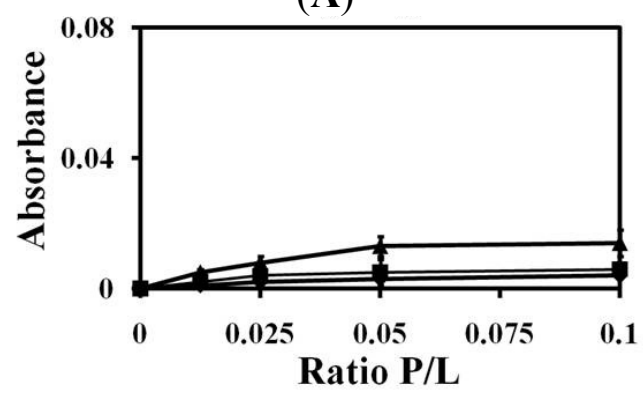

(C)

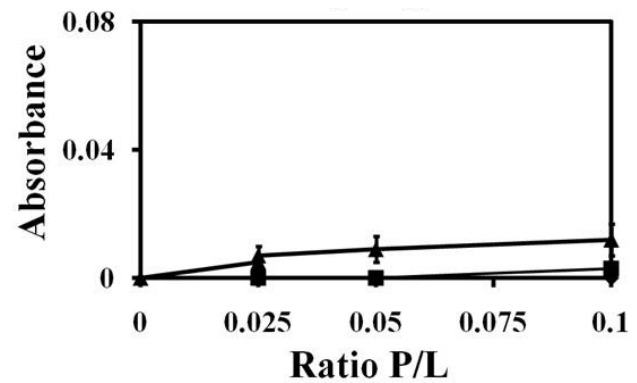

(B)



(D)

\subsection{Characterization of Trp Environment Using Fluorescence Spectroscopy}

To determine the mechanism behind $(\mathrm{KW})_{5}$-induced aggregation of eukaryotic liposomes and whether or not it is mediated by interactions with the zwitterionic eukaryotic membrane, Trp fluorescence blue shift and Trp quenching experiments were carried out to compare the membrane-binding affinities of the peptides. Peptide binding to lipid bilayers was examined by recording the Trp fluorescence emission spectra in the presence of vesicles composed of zwitterionic PC, PC:SM $(2: 1, w / w)$ and PC:CH $(2: 1, w / w)$ (Figure 2). Blue shift assay indicated that $(\mathrm{KW})_{3}$ and $(\mathrm{KW})_{4}$ did not strongly interact with any of the liposomes. Peptides that interact with negatively charged bacterial membranes through electrostatic interactions initially experience enhanced accumulation on the bilayer surface [26]. On the other hand, bilayer disruption strongly depends on the properties of lipids, as well as peptide charge/hydrophobicity. Both $(\mathrm{KW})_{3}$ and $(\mathrm{KW})_{4}$ did not strongly bind with $\mathrm{PC}$, PC:SM $(2: 1, w / w)$ or PC:CH $(2: 1, w / w)$ due to their zwitterionic character. Since PC membranes lack a net negative charge, cationic peptides cannot undergo electrostatic interactions with them. In contrast, $(\mathrm{KW})_{5}$ showed strong interactions towards all liposomes. Among the three liposomes, larger blue shift of $(\mathrm{KW})_{5}$ peptide suggests that its Trp side chain partitions more into PC: $\mathrm{CH}(7: 3, w / w)$ lipid bilayers. 
Figure 2. Blue shift in Trp fluorescence. Emission maxima from Trp in peptides in the presence of (A) PC; (B) PC:SM $(2: 1, w / w)$; and (C) PC:CH $(2: 1, w / w) .(\mathrm{KW})_{3}$ (diamonds), $(\mathrm{KW})_{4}$ (squares), $(\mathrm{KW})_{5}$ (triangles).

$\mathbf{A}$



B

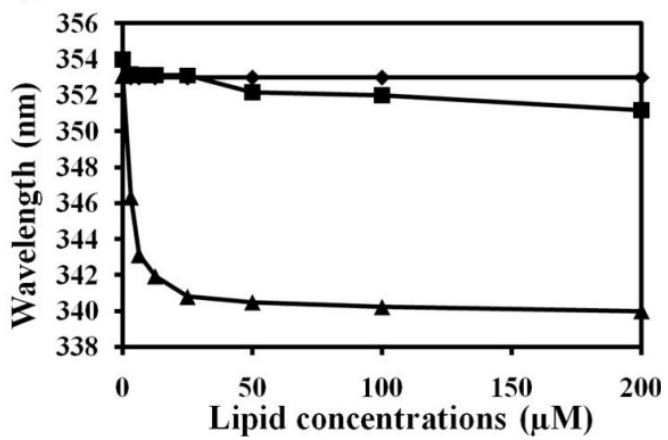

C

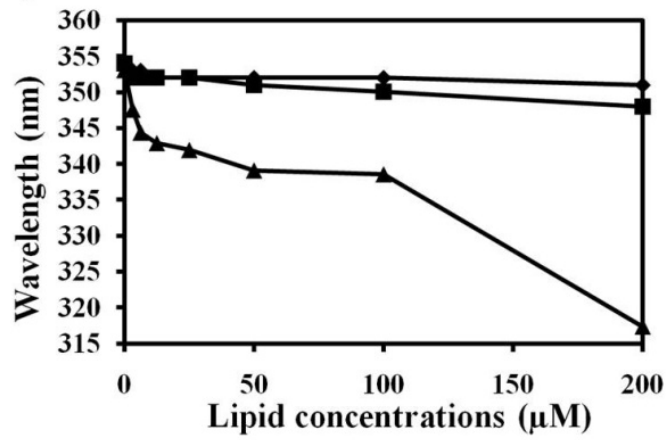

The accessibility of Trp residues into lipid bilayers can be determined by measuring the Stern-Volmer quenching constant for each peptide using soluble acrylamide as a quencher. In buffer, these peptides are almost completely accessible by acrylamide in aqueous solution [26]. The $K_{\mathrm{SV}}$ of $(\mathrm{KW})_{5}$ peptide was $1.7 \mathrm{M}^{-1}$, indicating that the Trp residues of $(\mathrm{KW})_{5}$ were more protected in the presence of PC:CH $(2: 1, w / w)$ vesicles than in PC or PC:SM $(2: 1, w / w)$ vesicles (Table 1$)$. This tendency was consistent with its potent aggregation activity towards eukaryotic liposomes. The lower Ksv values of vesicles composed of PC, PC:SM $(2: 1 w / w)$ and PC:CH $(2: 1 w / w)$ could be attributed to the hydrophobic effects and van der Waals forces that likely dominate the interactions between neutral lipids and the hydrophobic residues of $(\mathrm{KW})_{5}$.

Table 1. $K_{\text {Sv }}$ parameters of peptides at $2 \mu \mathrm{M}$ in PBS (pH 7.2) or in the presence of $200 \mu \mathrm{M}$ PC, $200 \mu \mathrm{M}$ PC:SM $(2: 1, w / w)$ and $200 \mu \mathrm{M}$ PC:CH $(2: 1 w / w)$ LUVs.

\begin{tabular}{cccc}
\hline \multirow{2}{*}{ Peptides } & \multicolumn{3}{c}{$\boldsymbol{K}_{\mathbf{S V}}\left(\mathbf{M}^{-\mathbf{1}}\right)^{\mathbf{a}}$} \\
\cline { 2 - 4 } & PC & PC:SM $(2: 1, w / w)$ & PC:CH $(2: 1 w / w)$ \\
\hline$(\mathrm{KW})_{3}$ & 7.5 & 7.1 & 6.8 \\
$(\mathrm{KW})_{4}$ & 7.2 & 6.8 & 6.2 \\
$(\mathrm{KW})_{5}$ & 3.2 & 2.5 & 1.7 \\
\hline
\end{tabular}

Notes: ${ }^{a} K_{\mathrm{SV}}$ is the Stern-Volmer constant; $K_{\mathrm{SV}}\left(\mathrm{M}^{-1}\right)$ was determined from the Stern-Volmer Equation $F_{0} / F_{1}=1+K_{\mathrm{SV}}(Q)$, where $Q$ is the concentration of quencher (acrylamide); Concentration of the quencher varied from 0.04 to $0.20 \mathrm{M}$. 


\subsection{Calcein Leakage}

Calcein leakage assay was performed to investigate interactions between AMPs and model liposomes [44]. We determined the ability of these peptides to cause leakage of entrapped calcein from PC, PC:SM $(2: 1 w / w)$ and PC:CH $(2: 1 w / w)$ vesicles, which mimic eukaryotic membranes. As shown in Figure $3,(\mathrm{KW})_{2}$ and $(\mathrm{KW})_{3}$ did not release calcein from any of the three liposomes, whereas $(\mathrm{KW})_{4}$ showed slight calcein leakage at a higher concentration of $64 \mu \mathrm{m}$. Generally, significant leakage is not observed from LUVs enriched in $\mathrm{PC}, \mathrm{CH}$ and $\mathrm{SM}$ by $(\mathrm{KW})_{2},(\mathrm{KW})_{3}$ or $(\mathrm{KW})_{4}$, which is consistent with their inability to induce aggregation/insertion in LUVs. In fact, Trp has a preference for the interfacial region of the lipid bilayer $[45,46]$. However, $(\mathrm{KW})_{2},(\mathrm{KW})_{3}$ and $(\mathrm{KW})_{4}$ did not deeply penetrate the hydrophobic region of the lipid bilayer, compared to $(\mathrm{KW})_{5}$ (Table 1). This may suggest that interactions of cationic peptides, such as $(\mathrm{KW})_{2},(\mathrm{KW})_{3}$ and $(\mathrm{KW})_{4}$, with neutral zwitterionic lipids are weakened due to repulsion between the cationic peptides and positively charged head group of amino choline in PC, thereby inhibiting the hydrophobic interactions between peptides and eukaryotic membranes. However, $(\mathrm{KW})_{5}$ showed eukaryotic membrane disruption ability due to two reasons. Firstly, $(\mathrm{KW})_{5}$ demonstrates weak aggregation in aqueous solution, which facilitates association of the peptide with liposomes by overcoming the repulsion between the peptide and lipid head group. Secondly, it has been described that increasing the number of Trp residues in AMPs naturally enhances membrane interactions and liposome leakage from $\mathrm{PC}$ or $\mathrm{PC}: \mathrm{CH}$; thus, $(\mathrm{KW})_{5}$ is more able to interact with the interfacial region of the bilayer [47]. Previous studies have also described AMP activity on PC vesicle through interfacial activity, resulting in binding, insertion and perturbation [48]. Interfacial activity is also displayed by peptides having sufficient hydrophobicity [49]. It has been clearly suggested that $(\mathrm{KW})_{5}$ peptides has higher hydrophobicity compared to $(\mathrm{KW})_{3}$ and $(\mathrm{KW})_{4}$. $(\mathrm{KW})_{5}$ peptide associates with the hydrocarbon core of neutral bilayers and localizes near the aqueous phase in zwitterionic model membranes (Table 1). This increased local density of $(\mathrm{KW})_{5}$, results in local disruption of lipid chains packing, leading to boundary disruption of lipid domains. The induced calcein release by $(\mathrm{KW})_{5}$ was consistent with this notion.

Figure 3. Percent leakage of calcein from (A) PC; (B) PC:SM $(2: 1, w / w)$; and (C) PC:CH $(2: 1, w / w)$ was measured for $25 \mathrm{~min}$ after the addition of various doses of peptide.

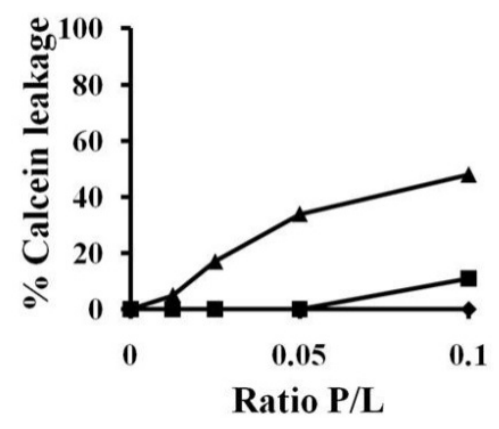

(A)

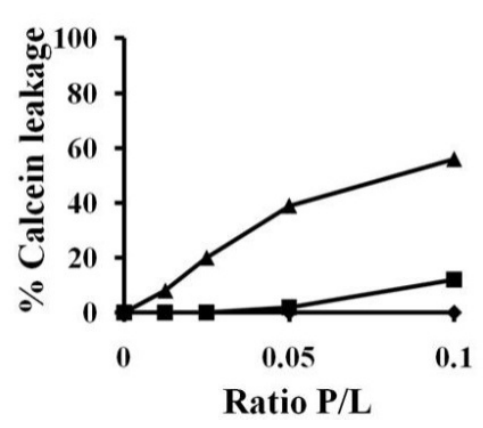

(B)

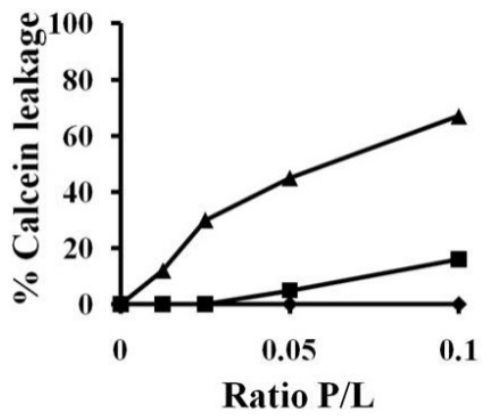

(C) 


\subsection{Circular Dichroism (CD)}

Conformational changes of peptides were investigated by CD spectroscopy. Dramatic changes in the CD spectra occurred when $(\mathrm{KW})_{5}$ was mixed with neutral lipids. In the presence of buffer, these peptides display random coil conformations [26]. When $(\mathrm{KW})_{5}$ was added to PC, PC:SM $(2: 1, w / w)$ and PC:CH $(2: 1, w / w)$ liposomes, larger changes in CD spectra were observed, especially in $\mathrm{PC}: \mathrm{CH}(2: 1, w / w)$. $(\mathrm{KW})_{5}$ shifted its minimum at $200 \mathrm{~nm}$ to $215 \mathrm{~nm}$ and gained a maximum at $195 \mathrm{~nm}$, indicating that an extended beta-sheet structure was formed (Figure 4). Similar behaviors have been observed with AMPs, such as $(\mathrm{RW})_{5}[22]$, suggesting that the high cost of partitioning peptide bonds into the membrane interface is a major driving force behind secondary structure formation in membrane environments [49-51]. Other studies also reported that peptides that are rich in aromatic residues bind well to the membrane interface [52]. In addition, previous information has shown that beta-sheet formation occurs upon induction of liposome aggregation by peptides [53]. Our CD measurement data also indicated that $(\mathrm{KW})_{5}$ underwent a conformational transition from a random coil [26] to a beta-sheet structure. This was consistent with its ability to disrupt lipid bilayers and promote aggregation of liposomes. Other AMPs, such as $(\mathrm{KW})_{2},(\mathrm{KW})_{3}$ and $(\mathrm{KW})_{4}$, did not strongly bind to zwitterionic PC, PC:SM $(2: 1, w / w)$ or PC:CH $(2: 1, w / w)$ vesicles, which was consistent with their lack of hemolytic activity in erythrocytes $[9,53,54]$. In this case, no specific interaction with cholesterol was observed, which indicates low toxicity towards normal mammalian cells [55]. Indeed, certain melittin analogues fail to adopt appreciable secondary structures in the presence of a mammalian mimetic membrane, which could be associated with their failure to bind to and permeabilize hRBCs through a toroidal-pore mechanism resembling hemolytic activity [56].

Figure 4. $\mathrm{CD}$ spectra for $(\mathrm{KW})_{4}$ and $(\mathrm{KW})_{5}$ peptides in the presence of $\mathrm{PC}$ (dashed line), PC:SM $(2: 1, w / w)$ (dashed dotted line) and PC:CH $(2: 1, w / w)$ (dotted). (A) $(\mathrm{KW})_{4}$ and (B) $(\mathrm{KW})_{5}$.

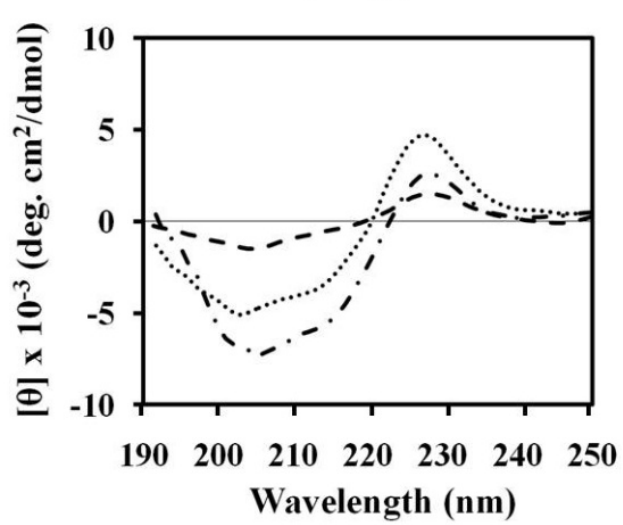

(A)

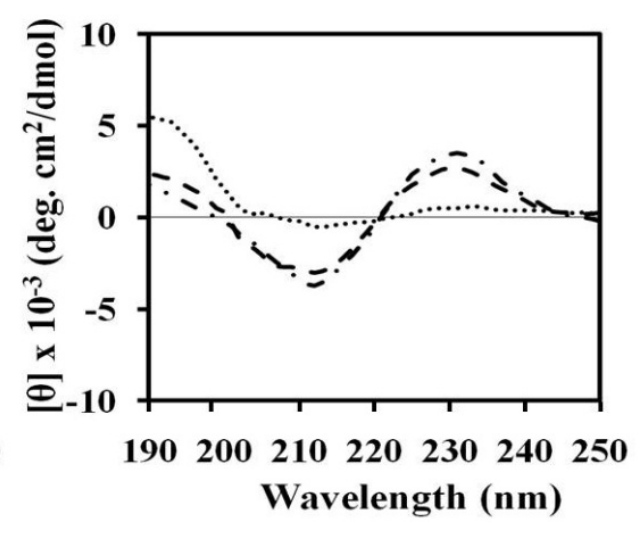

(B)

In this work, we confirmed that $(\mathrm{KW})_{5}$ induces membrane aggregation (or interaction) and membrane perturbation of vesicles composed of PC:CH $(2: 1, w / w), \operatorname{PC} / \mathrm{SM}(2: 1, w / w)$ and PC. We further demonstrated that $\mathrm{SM}, \mathrm{CH}$ and $\mathrm{PC}$ are not specifically required for the action of $(\mathrm{KW})_{5}$. Unlike $(\mathrm{KW})_{5}$, it was reported that some eukaryotic membrane active peptides are selective to the lipid composition of the model eukaryotic membrane [57,58]. Although the results of this study support the idea that the 
mechanism of $(\mathrm{KW})_{5}$ cytotoxicity involves membrane interactions/permeabilization along with liposome aggregation, further investigations are needed to explain how the liposome aggregation/fusion process is mediated.

\section{Experimental Section}

\subsection{Materials}

Rink amide 4-methylbenzhydrylamine resin, fluoren-9-ylmethoxycarbonyl (Fmoc) amino acids and other reagents for peptide synthesis were purchased from Calibochem-Novabiochem (La Jolla, CA, USA). $\mathrm{CH}$ from porcine liver, egg yolk $1-\alpha-\mathrm{PC}$ and SM were from Avanti Polar Lipids Co. (Alabaster, AL, USA). Calcein was obtained from Molecular Probes (Eugene, OR, USA). All other reagents were of analytical grade. Buffers were prepared using double distilled water (Millipore Co., Bedford, MA, USA).

\subsection{Peptide Synthesis and Purification}

Peptides were synthesized and purified as reported previously [26]. The purity of all peptides were found to be $>95 \%$.

\subsection{Preparation of Large Unilamellar Vesicles (LUVs)}

Large unilamellar vesicles (LUVs) were prepared by the freeze-thaw method [34,35]. Dry lipid films were resuspended in 1-2 $\mathrm{mL}$ of appropriate buffer by vortexing. LUVs were prepared by nine freeze-thaw cycles under liquid nitrogen and a water bath at $50{ }^{\circ} \mathrm{C}$. After preparation of vesicles, suspensions were extruded 14 times through a $0.2 \mu \mathrm{M}$ pore polycarbonate membrane. Lipid concentration was determined by standard phosphate assay [59].

\subsection{Liposome Aggregation}

Aggregation of lipid vesicles was monitored by visible absorbance measurements. The buffer used was PBS, pH 7.2. Peptides $(5,10,20$ and $40 \mu \mathrm{M})$ in PBS solutions were added to a suspension of $400 \mu \mathrm{M}$ LUVs consisting of PC, PC:SM $(2: 1, w / w)$ and PC:CH $(2: 1, w / w)$. Increased absorbance indicated aggregation of liposomes. Absorbance was measured at $405 \mathrm{~nm}$ using a microplate Autoreader before and after the addition of peptides [60].

\subsection{Trp Fluorescence and Acrylamide Quenching Assay}

Fluorescence emission spectra of Trp residue in peptides were monitored in the presence of PC, PC:SM $(2: 1, w / w)$ and PC:CH $(2: 1, w / w)$ LUVs. In these fluorescence studies, LUVs were used to minimize differential light scattering effects [61]. Trp fluorescence measurements were carried out using a spectrofluorometer. Each peptide was added to $1 \mathrm{ml}$ of $200 \mu \mathrm{M}$ liposomes, and the peptide:liposome mixture (a molar ratio of $1: 100$ ) was allowed to interact at $25^{\circ} \mathrm{C}$ for $10 \mathrm{~min}$. Fluorescence was measured at an excited wavelength of $280 \mathrm{~nm}$ an emission wavelength ranging from 300 to $400 \mathrm{~nm}$. 
Fluorescence quenching experiments were conducted using acrylamide as a quencher. The acrylamide concentration in the cuvette ranged from 0.04 to $0.20 \mathrm{M}$. The effect of acrylamide on the fluorescence of each peptide was analyzed using a Stern-Volmer Equation:

$$
F_{0} / F=1+K_{\mathrm{SV}}(Q)
$$

where $F_{0}$ and $F$ represent the fluorescence intensities in the absence and presence of acrylamide, respectively, $K_{\mathrm{SV}}$ is the Stern-Volmer quenching constant and $(Q)$ is the concentration of acrylamide.

\subsection{Calcein Leakage from Liposomes}

Calcein-entrapped LUVs composed of PC, PC:SM $(2: 1, w / w)$ and PC:CH $(2: 1, w / w)$ were prepared by vortexing the dried lipid in a dye buffer solution (70 mM calcein, PBS, $\mathrm{pH} 7.4)$. The suspension was freeze-thawed in liquid nitrogen for nine cycles, after which the calcein-entrapped vesicles were separated from free calcein by gel filtration chromatography on a Sephadex G-50 column. Entrapped LUVs in a suspension containing $100 \mu \mathrm{M}$ lipids were then incubated with various concentrations of peptide $(0.625-10 \mu \mathrm{M})$ for $25 \mathrm{~min}$. The fluorescence of released calcein was assessed using a spectrofluorometer at an excitation wavelength of $480 \mathrm{~nm}$ and an emission wavelength of $520 \mathrm{~nm}$. Complete (100\%) release was achieved by the addition of $0.1 \%$ Triton X-100. Spontaneous leakage was determined to be negligible. All experiments were conducted at $25{ }^{\circ} \mathrm{C}$, and the apparent percentage of released calcein was calculated according to the following Equation [62]:

$$
\text { Release }(\%)=100 \times\left(F-F_{\mathrm{o}}\right) /\left(F_{\mathrm{t}}-F_{\mathrm{o}}\right)
$$

where $F$ and $F_{\mathrm{t}}$ represent the fluorescence intensity before and after the addition of detergent, respectively, and $F_{\mathrm{o}}$ represents the fluorescence of intact vesicles.

\subsection{Circular Dichroism (CD) Spectroscopy}

The CD spectra were recorded on a Jasco 810 spectropolarimeter (Jasco, Tokyo, Japan) equipped with a temperature control unit using a $0.1-\mathrm{cm}$ path-length quartz cell at $25^{\circ} \mathrm{C}$ between 190 and $250 \mathrm{~nm}$. The CD spectra were measured for peptide samples $(50 \mu \mathrm{M})$ that were dissolved in PBS ( $\mathrm{pH} 7.2)$ containing $1 \mathrm{mM} \mathrm{PC}, 1 \mathrm{mM}$ PC:SM $(2: 1, w / w)$ vesicles or $1 \mathrm{mM} \mathrm{PC:CH}(2: 1, w / w)$ vesicles. CD data represent the average value from three separate recordings, with four scans per sample. All CD spectra shown had corresponding peptide-free solvent baselines subtracted. The results are expressed in terms of molar residue $\mathrm{CD}$.

\section{Conclusions}

Among $(\mathrm{KW})_{n}$ peptides, only $(\mathrm{KW})_{5}$ induced permeation/aggregation of PC vesicles alone or in combination with $\mathrm{CH}$ or SM. Eukaryotic membrane aggregation induced by $(\mathrm{KW})_{5}$ was dependent on both the presence of a more hydrophobic patch, as well as its ability to self-aggregate in aqueous solution compared to $(\mathrm{KW})_{4}$. Therefore, $(\mathrm{KW})_{5}$ is not a selective peptide against bacterial membranes compared to $(\mathrm{KW})_{4}$. 


\section{Acknowledgements}

This work was supported by a National Research Foundation of Korea (NRF) grant funded by the Korea government (MEST) (No. 2011-0017532) and the Human Resource Training Project for Regional Innovation.

\section{References}

1. Hoskin, D.W.; Ramamoorthy, A. Studies on anticancer activities of antimicrobial peptides. Biochim. Biophys. Acta 2008, 1778, 357-375.

2. Dürr, U.H.N.; Sudheendra, U.S.; Ramamoorthy, A. LL-37, the only human member of the cathelicidin family of antimicrobial peptides. Biochim. Biophys. Acta 2006, 1758, 1408-1425.

3. Dhople V.; Krukemeyer, A.; Ramamoorthy, A. The human beta-defensin-3, an antibacterial peptide with multiple biological functions. Biochim. Biophys. Acta 2006, 1758, 1499-1512.

4. Abraham, T.; Lewis, R.N.; Hodges, R.S.; McElhaney, R.N. Isothermal titration calorimetry studies of the binding of the antimicrobial peptide gramicidin $\mathrm{S}$ to phospholipid bilayer membranes. Biochemistry 2005, 44, 11279-11285.

5. Verkleij, A.J.; Zwaal, R.F.; Roelofsen, B.; Comfurius, P.; Kastelijn, D.; van Deenen, L.L. The asymmetric distribution of phospholipids in the human red cell membrane. A combined study using phospholipases and freeze-etch electron microscopy. Biochim. Biophys. Acta 1973, 323, 178-193.

6. Vogel, H.J.; Schibli, D.J.; Jing, W.; Lohmeier-Vogel, E.M.; Epand, R.F.; Epand, R.M. Towards a structure-function analysis of bovine lactoferricin and related tryptophan- and arginine-containing peptides. Biochem. Cell Biol. 2002, 80, 49-63.

7. Matsuzaki, K. Why and how are peptide-lipid interactions utilized for self-defense? Magainins and tachyplesins as archetypes. Biochim. Biophys. Acta 1999, 1462, 1-10.

8. Lugtenberg, B.; van Alphen, L. Molecular architecture and functioning of the outer membrane of Escherichia coli and other gram-negative bacteria. Biochim. Biophys. Acta 1983, 737, 51-115.

9. Glukhov, E.; Stark, M.; Burrows, L.L.; Deber, C.M. Basis for selectivity of cationic antimicrobial peptides for bacterial versus mammalian membranes. J. Biol. Chem. 2005, 280, 33960-33967.

10. Epand, R.F.; Schmitt, M.A.; Gellman, S.H.; Epand, R.M. Role of membrane lipids in the mechanism of bacterial species selective toxicity by two alpha/beta-antimicrobial peptides. Biochim. Biophys. Acta 2006, 1758, 1343-1350.

11. Shai, Y.; Fox, J.; Caratsch, C.; Shih, Y.L.; Edwards, C.; Lazarovici, P. Sequencing and synthesis of pardaxin, a polypeptide from the Red Sea Moses sole with ionophore activity. FEBS Lett. 1988, 242, 161-166.

12. Oren, Z.; Shai, Y. Selective lysis of bacteria, but not mammalian cells by diastereomers of melittin: Structure-function study. Biochemistry 1997, 36, 1826-1835.

13. Johansson, J.; Gudmundsson, G.H.; Rottenberg, M.E.; Berndt, K.D.; Agerberth, B. Conformation-dependent antibacterial activity of the naturally occurring human peptide LL-37. J. Biol. Chem. 1998, 273, 3718-3724.

14. Chen, Y.; Mant, C.T.; Farmer, S.W.; Hancock, R.E.; Vasil, M.L.; Hodges, R.S. Rational design of alpha-helical antimicrobial peptides with enhanced activities and specificity/therapeutic index. J. Biol. Chem. 2005, 280, 12316-12329. 
15. Sengupta, D.; Leontiadou, H.; Mark, A.E.; Marrink, S.J. Toroidal pores formed by antimicrobial peptides show significant disorder. Biochim. Biophys. Acta 2008, 1778, 2308-2317.

16. Melo, M.N.; Ferre, R.; Castanho, M.A. Antimicrobial peptides: Linking partition, activity and high membrane-bound concentrations. Nat. Rev. Microbiol. 2009, 7, 245-250.

17. Zhu, W.L.; Nan, Y.H.; Hahm, K.S.; Shin, S.Y. Cell selectivity of an antimicrobial peptide melittin diastereomer with D-amino acid in the leucine zipper sequence. J. Biochem. Mol. Biol. 2007, 40, 1090-1094.

18. Matsuzaki, K. Control of cell selectivity of antimicrobial peptides. Biochim. Biophys. Acta 2009, 1788, 1687-1692.

19. Huang, Y.; Huang, J.; Chen, Y. Alpha-helical cationic antimicrobial peptides: Relationships of structure and function. Protein Cell 2010, 1, 143-152.

20. Wang, P.; Nan, Y.H.; Yang, S.T.; Kang, S.W.; Kim, Y.; Park, I.S.; Hahm, K.S.; Shin, S.Y. Cell selectivity and anti-inflammatory activity of a Leu/Lys-rich alpha-helical model antimicrobial peptide and its diastereomeric peptides. Peptides 2010, 31, 1251-1261.

21. Strom, M.B.; Haug, B.E.; Sker, M.L.; Stensen, W.; Stiberg, T.; Svendsen, J.S. The Pharmacophore of short cationic antibacterial peptides. J. Med. Chem. 2003, 46, 1567-1570.

22. Liu, Z.; Brady, A.; Young, A.; Rasimick, B.; Chen, K.; Zhou, C.; Kallenbach, N.R. Length effects in antimicrobial peptides of the (RW) ${ }_{n}$ series. Antimicrob. Agents Chemother. 2007, 51, 597-603.

23. Strom, M.B.; Rekdal, O.; Svendsen, J.S. Antimicrobial activity of short arginine- and tryptophan-rich peptides. J. Pept. Sci. 2002, 8, 431-437.

24. Jing, W.; Hunter, H.N.; Hagel, J.; Vogel, H.J. The structure of the antimicrobial peptide Ac-RRWWRF-NH $\mathrm{N}_{2}$ bound to micelles and its interactions with phospholipid bilayers. J. Pept. Res. 2003, 61, 219-229.

25. Dathe, M.; Nikolenko, H.; Klose, J.; Bienert, M. Cyclization increases the antimicrobial activity and selectivity of arginine- and tryptophan- containing hexapeptides. Biochemistry 2004, 43, 9140-9150.

26. Gopal, R.; Seo, C.H.; Song, P.I.; Park, Y. Effect of repetitive lysine-tryptophan motifs on the bactericidal activity of antimicrobial peptides. Amino Acids 2012, [Epub ahead of print].

27. Chen, Y.; Guarnieri, M.T.; Vasil, A.I.; Vasil, M.L.; Mant, C.T.; Hodges, R.S. Role of peptide hydrophobicity in the mechanism of action of alpha-helical antimicrobial peptides. Antimicrob. Agents Chemother. 2007, 51, 1398-1406.

28. Ramamoorthy, A.; Lee, D.K.; Narasimhaswamy, T.; Nanga R.P.R. Cholesterol reduces pardaxin's dynamics - a barrel — stave mechanism of membrane disruption investigated by solid-state NMR. Biochim. Biophys. Acta 2010, 1798, 223-227.

29. McHenry, A.J.; Sciacca, M.F.M.; Brender, J.R.; Ramamoorthy, A. Does cholesterol suppress the antimicrobial peptide induced disruption of lipid raft containing membranes? Biochim. Biophys. Acta 2012, 1818, 3019-3024.

30. Bhattacharjya, S.; Ramamoorthy, A. Multifunctional host defense peptides: Functional and mechanistic insights from NMR structures of potent antimicrobial peptides. FEBS J. 2009, 276, 6465-6473.

31. Ramamoorthy, A. Beyond NMR spectra of antimicrobial peptides: Dynamical images at atomic resolution and functional insights. Solid State Nucl. Magn. Reson. 2009, 35, 201-207. 
32. Gottler, L.M.; Ramamoorthy, A. Structure, membrane orientation, mechanism and function of pexiganan-A highly potent antimicrobial peptide designed from magainin. Biochim. Biophys. Acta 2009, 1788, 1680-1686.

33. March, E.N.; Buer, B.C.; Ramamoorthy, A. Fluorine-A new element in the design of membrane-active peptides. Mol. Biosyst. 2009, 5, 1143-1147.

34. Matsuyama, K.; Natori, S. Mode of action of sapecin, a novel antibacterial protein of Sarcophaga peregrina (flesh fly). J. Biochem. 1990, 108, 128-132.

35. Dhople, V.M.; Nagaraj, R. Generation of analogs having potent antimicrobial and hemolytic activities with minimal changes from an inactive 16-residue peptide corresponding to the helical region of Staphylococcus aureus $\delta$-toxin. Protein Eng.1995, 8, 315-318.

36. Blondelle, S.E.; Lohner, K.; Aguilar, M. Lipid induced conformation and lipid-binding properties of cytolytic and antimicrobial peptides: Determination and biological specificity. Biochim. Biophys. Acta 1999, 1462, 89-108.

37. Som, A.; Vemparala, S.; Ivanov, I.; Tew, G.N. Synthetic mimics of antimicrobial peptides. Biopolymers 2008, 90, 83-93.

38. Gopal, R.; Park, S.C.; Ha, K.J.; Cho, S.J.; Kim, S.W.; Song, P.I.; Nah, J.W.; Park, Y.; Hahm, K.S. Effect of leucine and lysine substitution on the antimicrobial activity and evaluation of the mechanism of the HPA3NT3 analog peptide. J. Pept. Sci. 2009, 15, 589-594.

39. Rathinakumar, R.; Walkenhorst, W.F.; Wimley, W.C. Broad-spectrum antimicrobial peptides by rational combinatorial design and high-throughput screening: The importance of interfacial activity. J. Am. Chem. Soc. 2009, 131, 7609-7617.

40. Zhu, W.L.; Shin, S.Y. Effects of dimerization of the cell-penetrating peptide Tat analog on antimicrobial activity and mechanism of bactericidal action. J. Pept. Sci. 2009, 15, 345-352.

41. Torrent, M.; de la Torre, B.G.; Nogués, V.M.; Andreu, D.; Boix, E. Bactericidal and membrane disruption activities of the eosinophil cationic protein are largely retained in an N-terminal fragment. Biochem. J. 2009, 421, 425-434.

42. Javadpour, M.M.; Barkley, M.D. Self-assembly of designed antimicrobial peptides in solution and micelles. Biochemistry 1997, 36, 9540-9549.

43. Feder, R.; Dagan, A.; Mor, A. Structure-activity relationship study of antimicrobial dermaseptin S4 showing the consequences of peptide oligomerization on selective cytotoxicity. J. Biol. Chem. 2000, 275, 4230-4238.

44. Chongsiriwatana, N.P.; Barron, A.E. Comparing bacterial membrane interactions of antimicrobial peptides and their mimics. Methods Mol. Biol. 2010, 618, 171-182.

45. Subbalakshmi, C.; Krishnakumari, V.; Sitaram, N.; Nagaraj, R. Interaction of indolicidin, a 13-residue peptide rich in tryptophan and proline and its analogues with model membranes. J. Biosci. 1998, 23, 9-13.

46. Takahashi, D.; Shukla, S.K.; Prakash, O.; Zhang, G. Structural determinants of host defense peptides for antimicrobial activity and target cell selectivity. Biochimie 2010, 92, 1236-1241.

47. Schmidtchen, A.; Pasupuleti, M.; Mörgelin, M.; Davoudi, M.; Alenfall, J.; Chalupka, A.; Malmsten, M. Boosting antimicrobial peptides by hydrophobic oligopeptide end tags. J. Biol. Chem. 2009, 284, 17584-17594. 
48. William, C.W. Describing the mechanism of antimicrobial peptide action with the interfacial activity model. ACS Chem. Biol. 2010, 5, 905-917.

49. Wimley, W.C.; Hristova, K.; Ladokhin, A.S.; Silvestro, L.; Axelsen, P.H.; White, S.H. Folding of beta-sheet membrane proteins: A hydrophobic hexapeptide model. J. Mol. Biol. 1998, 277, 1091-1110.

50. Ladokhin, A.S.; White, S.H. Folding of amphipathic alpha-helices on membranes: Energetics of helix formation by melittin. J. Mol. Biol. 1999, 285, 1363-1369.

51. Wimley, W.C.; White, S.H. Experimentally determined hydrophobicity scale for proteins at membrane interfaces. Nat. Struc. Biol. 1996, 3, 842-848.

52. Thennarasu, S.; Huang, R.; Lee, D.K.; Yang, P.; Maloy, L.; Chen, Z.; Ramamoorthy, A. Limiting an antimicrobial peptide to the lipid-water interface enhances its bacterial membrane selectivity: A case study of MSI-367. Biochemistry 2010, 49, 10595-10605.

53. Andrä, J.; Monreal, D.; Martinez de Tejada, G.; Olak, C.; Brezesinski, G.; Gomez, S.S.; Goldmann, T.; Bartels, R.; Brandenburg, K.; Moriyon, I. Rationale for the design of shortened derivatives of the NK-lysin-derived antimicrobial peptide NK-2 with improved activity against Gram-negative pathogens. J. Biol. Chem. 2007, 282, 14719-14728.

54. Hawrani, A.; Howe, R.A.; Walsh, T.R.; Dempsey, C.E. Origin of low mammalian cell toxicity in a class of highly active antimicrobial amphipathic helical peptides. J. Biol. Chem. 2008, 283, 18636-18645.

55. Zhao, H.; Sood, R.; Jutila, A.; Bose, S.; Fimland, G.; Nissen-Meyer, J.; Kinnunen, P.K. Interaction of the antimicrobial peptide pheromone Plantaricin A with model membranes: Implications for a novel mechanism of action. Biochim. Biophys. Acta 2006, 1758, 1461-1474.

56. Pandey, B.K.; Ahmad, A.; Asthana, N.; Azmi, S.; Srivastava, R.M.; Srivastava, S.; Verma, R.; Vishwakarma, A.L.; Ghosh, J.K. Cell-selective lysis by novel analogues of melittin against human red blood cells and Escherichia coli. Biochemistry 2010, 49, 7920-7929.

57. Lee, J.K.; Park, S.C.; Hahm, K.S.; Park, Y. Antimicrobial HPA3NT3 peptide analogs: Placement of aromatic rings and positive charges are key determinants for cell selectivity and mechanism of action. Biochim. Biophys. Acta 2012, 1828, 443-454.

58. Brender, J.R.; McHenry, A.J.; Ramamoorthy, A. Does cholesterol role in the bacterial selectivity of antimicrobial peptides? Front. Immunol. 2012, 3, 195-198.

59. Steward, J.C. Colorimetric determination of phospholipids with ammonium ferrothiocyanate. Anal. Biochem. 1980, 104, 10-14.

60. Oren, Z.; Lerman, J.C.; Gudmundsson, G.H.; Agerberth, B.; Shai, Y. Structure and organization of the human antimicrobial peptide LL-37 in phospholipid membranes: Relevance to the molecular basis for its non-cell-selective activity. Biochem. J. 1999, 341, 501-513.

61. Mao, D.; Wallace, B.A. Differential light scattering and adsorption flattening optical effects are minimal in the circular dichroism spectra of small unilamellar vesicles. Biochemistry 1984, 23, 2667-2673.

62. Matsuzaki, K.; Sugishita, K.; Miyajima, K. Interactions of an antimicrobial peptide, magainin 2, with lipopolysaccharide containing liposomes as a model for outer membranes of gram-negative bacteria. FEBS Lett. 1999, 449, 221-224.

(C) 2013 by the authors; licensee MDPI, Basel, Switzerland. This article is an open access article distributed under the terms and conditions of the Creative Commons Attribution license (http://creativecommons.org/licenses/by/3.0/). 